\title{
DEEP IMPACT: WORKING PROPERTIES FOR THE TARGET NUCLEUS - COMET 9P/TEMPEL 1
}

\author{
MICHAEL J.S. BELTON ${ }^{1}$, KAREN J. MEECH ${ }^{2}$, MICHAEL F. A'HEARN ${ }^{3}$, \\ OLIVIER GROUSSIN ${ }^{3}$, LUCY MCFADDEN ${ }^{3}$, CAREY LISSE $^{3}$, \\ YANGA R. FERNÁNDEZ ${ }^{2}$, JANA PITTICHOVÁ ${ }^{2}$, HENRY HSIEH ${ }^{2}$ \\ JOCHEN KISSEL ${ }^{4}$, KENNETH KLAASEN $^{5}$, PHILIPPE LAMY ${ }^{6}$, DINA PRIALNIK ${ }^{7}$, \\ JESSICA SUNSHINE ${ }^{8}$, PETER THOMAS ${ }^{9}$, and IMRE TOTH ${ }^{10}$ \\ ${ }^{1}$ Belton Space Exploration Initiatives, LLC, Tucson, AZ, USA \\ ${ }^{2}$ Institute for Astronomy, University of Hawaii, Honolulu, HI, USA \\ ${ }^{3}$ University of Maryland, College Park, MD, USA \\ ${ }^{4}$ Max-Planck-Institut für Sonnensystemforschung, Katlenburg-Lindau, Germany \\ ${ }^{5}$ Jet Propulsion laboratory, Pasadena, CA, USA \\ ${ }^{6}$ Laboratoire d'Astronomie Spatiale CNRS, Marseille, France \\ ${ }^{7}$ Dept. of Geophysics and Planetary Sciences, Tel Aviv University, Israel \\ ${ }^{8}$ Science Applications International Corporation, Chantilly, VA, USA \\ ${ }^{9}$ Center for Radiophysics and Space Research, Cornell University, Ithaca, NY, USA \\ ${ }^{10}$ Konkoly Observatory, Budapest, Hungary
}

(Received August 21, 2004, Revised December 14, 2004.) 
Suggested running head: The nucleus of comet 9P/Tempel 1

Editorial correspondence to:

Dr. Michael Belton

Belton Space Exploration Initiatives, LLC

430 S.Randolph Way

Tucson, AZ, 85716

USA

Phone: $\quad 520-795-6220$

Fax: $\quad$ 520-795-6220

Email:_ michaelbelton@beltonspace.com

Key words: Comets, Space Missions, Nucleus, 9P/Tempel 1 
Abstract: In 1998, Comet 9P/Tempel 1 was chosen as the target of the Deep Impact mission (A'Hearn et $a l ., 2004)$ even though very little was known about its physical properties. Efforts where immediately begun to improve this situation by the Deep Impact Science Team leading to the founding of a world wide observing campaign (Meech et al., 2004a). This campaign has already produced a great deal of information on the global properties of the comet's nucleus (summarized in Table I) that is vital to the planning and the assessment of the chances of success at the impact and encounter. Since the mission was begun the successful encounters of the Deep Space 1 spacecraft at Comet 19P/Borrelly and the Stardust spacecraft at Comet $81 \mathrm{P} /$ Wild 2 have occurred yielding new information on the state of the nuclei of these two comets. This information, together with earlier results on the nucleus of comet 1P/Halley from the European Space Agency's Giotto, the Soviet Vega mission and various ground-based observational and theoretical studies, is used as a basis for conjectures on the morphological, geological, mechanical, and compositional properties of the surface and subsurface that Deep Impact may find at 9P/Tempel 1. We adopt the following working values (circa December 2004) for the nucleus parameters of prime importance to Deep Impact as follows: mean effective radius $=3.25 \pm 0.2 \mathrm{~km}$, shape - irregular tri-axial ellipsoid with $\mathrm{a} / \mathrm{b}=$ $3.2 \pm 0.4$ and overall dimensions of $\sim 14.4 \times 4.4 \times 4.4 \mathrm{~km}$, principal axis rotation with period $=41.85 \pm 0.1$ hr, pole directions $(\mathrm{RA}$, Dec, J2000) $=46 \pm 10,73 \pm 10 \operatorname{deg}($ Pole 1) or $287 \pm 14,16.5 \pm 10 \operatorname{deg}($ Pole 2) (the two poles are photometrically, but not geometrically, equivalent), Kron - Cousins (V-R) color $=0.56 \pm$ 0.02 , V-band geometric albedo $=0.04 \pm 0.01$, R-band geometric albedo $=0.05 \pm 0.01$, R-band $\mathrm{H}(1,1,0)=$ $14.441 \pm 0.067$, and mass $\sim 7 \times 10^{13} \mathrm{~kg}$ assuming a bulk density of $500 \mathrm{~kg} \cdot \mathrm{m}^{-3}$. As these are working values, i.e., based on preliminary analyses, it is expected that adjustments to their values may be made before encounter as improved estimates become available through further analysis of the large database being made available by the Deep Impact observing campaign. Given the parameters listed above the impact will occur in an environment where the local gravity is estimated at $0.027-0.04 \mathrm{~cm} . \mathrm{s}^{-2}$ and the escape velocity between 1.4 and $2 \mathrm{~m} . \mathrm{s}^{-1}$. For both of the rotation poles found here, the Deep Impact spacecraft on approach to encounter will find the rotation axis close to the plane of the sky (aspect angles 82.2 and $69.7 \mathrm{deg}$. for pole 1 and 2 respectively). However, until the rotation period estimate is substantially improved, it will remain uncertain whether the impactor will collide with the broadside or the ends of the nucleus. 


\section{Introduction.}

Comet 9P/Tempel 1 was chosen from a short list of possible targets in 1998, not because a great deal was known about the properties of its nucleus, but because of its orbital properties (Yeomans et al., 2004). As described by A'Hearn et al. (2004), the comet provided an acceptable launch date, an acceptable solar phase angle on approach, and an impact at a time when the comet was observable from the vicinity of the Earth. All that was known about the nucleus properties at the time it was chosen was that it appeared to be a typical Jupiter family comet with perhaps only a small fraction of its surface active (A'Hearn et al., 1995). Later, as a consequence of Hubble Space Telescope (HST) data taken in December 1997 (Lamy et al., 2001), information on the effective radius of the nucleus $(\sim 3 \mathrm{~km})$ became available. There was also an indication that the rotational period was about a day or longer. As we shall develop in this paper, preliminary results from the Deep Impact observing campaign (Meech et al., 2004a) now indicate an R-band geometric albedo, phase law, and light curve modulations for 9P/Tempel 1 that are substantial improvements over what had to be assumed at the beginning of the project. Although information available in 1998 was sufficient to make 9P/Tempel 1 an acceptable target, it was clear that a far more precise body of information would be needed to both aid in the technical development of the mission (particularly in the areas of navigation of the impactor and the assessment of the hazard due to dust in the coma) and to help interpret the data acquired during the encounter in the immediate post-impact period. Deep Impact is a fast $(10.2 \mathrm{~km} / \mathrm{sec})$ dual spacecraft flyby/impactor mission and during the short time interval when the nucleus is spatially resolved its instruments will 
only view the nucleus at a limited number of viewing angles and rotational phases (Klaasen et al., 2004). Optimum mission planning greatly benefits from prior knowledge of the nucleus shape, spin state and global surface properties. To provide estimates of these, the Deep Impact science team organized an observing campaign that included investigations with some of the most powerful telescopes, both on the ground and in space. Since the initiation of the project, this campaign has, as described in Meech et al. (2004a), produced high quality ground-based data on the nucleus and its coma at all apparitions of the comet from 1999 through 2004. It has also utilized powerful facilities such as the Keck telescope (Fernández et al., 2003), the European Southern Observatory Very Large Telescope, the Hubble Space Telescope, the Spitzer Space Telescope infrared facility (Lisse et al., 2004a), several 4m telescopes, and the University of Hawaii 2.2m telescope (Fernández et al., 2003).

\section{Global Properties of the Nucleus:}

Table I contains values of parameters that we have adopted as 'working' estimates of the global properties of the nucleus of 9P/Tempel 1 for the purposes of contemporary mission planning. Because the analysis of the large data set obtained during the Deep Impact observing campaign is still incomplete, some of the values in this table can be expected to undergo revision as the analysis continues. However, at this stage most of the parameters are sufficiently well established to provide a useful and quantitative picture of the nucleus. 


\subsection{MEAN DENSITY.}

Prominent in Table I is the uncertainty in the estimate of the mean bulk density of the nucleus, which we take to be $500 \pm 400 \mathrm{~kg} \cdot \mathrm{m}^{-3}$. Even after three space encounters with cometary nuclei this parameter remains beyond our ability to measure directly. Indirect attempts involving the analysis of orbital motion in response to reaction forces applied as the result of cometary activity (Rickman (1986, 1989), Sagdeev et al. (1987) and (1989) or, e.g., in the specific case of comet $1 \mathrm{P} /$ Halley with a correct rotational model by Samarasinha and Belton (1995), tidal forces (e.g., the case of Comet D/Shoemaker-Levy 9 by Asphaug and Benz, 1996), and rotational stability (Jewitt and Meech, 1988; Lowry and Weissman, 2003) provide confidence that a typical mean bulk density lies somewhere between 100 and $1000 \mathrm{~kg} \cdot \mathrm{m}^{-3}$. In addition, an estimate of $300 \mathrm{~kg} \cdot \mathrm{m}^{-3}$ for the mean bulk density is provided by the interstellar aggregate dust model (Greenberg et al., 1995). In a recent review, apparently following work by Skorov and Rickman (1999), Weissman et al. (2004) recommend a bulk density in the range 500 to $1200 \mathrm{~kg} \cdot \mathrm{m}^{-3}$; our assessment, which is more strongly weighted by the numerical experiments of Samarasinha and Belton (1995), overlaps this range. During encounter Deep Impact will attempt to measure the mass and, by implication, the mean density of 9P/Tempel 1 by following the trajectories of any pieces of large ejecta or clumps of smaller ejecta that may be released in to ballistic orbits by the impact. The dimensions and shape of the nucleus will also be determined. 


\subsection{DIMENSIONS OF THE NUCLEUS.}

Investigations of the effective radius and shape of the nucleus were the focus of work by Weissman et al. (1999), Lowry et al. (1999), Lamy et al. (2001), and later by Fernández et al. (2003), the latter employing the Keck and University of Hawaii $2.2 \mathrm{~m}$ telescope on Mauna Kea. These early results are summarized in Lamy et al. (2004) who suggest an effective radius of $3.1 \mathrm{~km}$, a minimum axial ratio of 1.40 and rotation period of $41 \mathrm{hr}$. More recently, observations in the thermal infra-red have been acquired in support of Deep Impact from the newly launched Spitzer Space Telescope (Lisse et al., 2004a) and results, based on thermal measurements, are now available. In addition, the Deep Impact ground-based campaign (Meech et al., 2004a) has produced a large database of photometric R-band and visual observations that characterize the comet in all phases of its orbit. This has allowed accurate values for the absolute magnitude, phase law coefficients, color, pole direction and axial ratio to be determined.

The observations of Weissman et al. (1999) and the observations of Lamy et al. (2001) using the HST were taken when the comet was near aphelion (4.74 AU). The authors, in their interpretation of these observations were required to make the assumption that it was inactive at such heliocentric distances in order to determine an effective radius for the nucleus. Today this assumption is suspect and should be justified in each application. At least one other Jupiter family comet, 2P/Encke, has been shown to be active at its aphelion (4.09 AU) even in the absence of a detectable coma (Meech et al., 2001; Belton et al., 2005). In this paper, we justify the use of this assumption by appealing to the behavior of 9P/Tempel 1's R-band light curve beyond 4 AU. In Fig. 1 
we show a light curve based on R-band data obtained during the Deep Impact observing campaign that covers both its active phase near perihelion and its, possibly, inactive phase near aphelion. The steep brightening at heliocentric distances less than $3.5 \mathrm{AU}$ contrasts with the behavior beyond $4 \mathrm{AU}$ where large rotational variations are seen ( $c f$. Figs. 2 and 3 for more detail) and that are superposed on a mean value that is essentially independent of heliocentric distance. While some active contribution cannot be discounted, the observed behavior is consistent with the assumption that the light reflected off the nucleus is the dominant contributor to the light curve. This should be compared with the quite different behavior seen in 2P/Encke at $4 \mathrm{AU}$ where activity contributions range over several magnitudes and the nucleus component can be shown to be usually only a few 10's of percent of the total light (Meech et al., 2001; Belton et al., 2005). With the assumption that 9P/Tempel 1 was inactive at $4.48 \mathrm{AU}$, Lamy et al., (2001) found a mean radius of $3.06 \pm 0.25 \mathrm{~km}$ with the additional assumptions that the geometric albedo was 0.04 and a linear phase coefficient of $0.04 \mathrm{mag} / \mathrm{deg}$ applied. In these observations, only a fraction of the rotational variation of the light curve was observed, which made it difficult to ensure how well this result applies to an estimate of a mean effective radius (i.e., averaged over a complete rotational cycle). An earlier study by Lowry et al. (1999) suggested an effective radius of $2.3 \pm 0.5 \mathrm{~km}$ under similar albedo and phase coefficient assumptions, but in this case coma was definitely present and the result is much less certain.

The Deep Impact related investigations of Fernández et al. (2003) focused on thermal infrared and R-band observations from co-located telescopes in order to remove the necessity of making assumptions about the geometric albedo and phase coefficient 
when deducing the effective radius of the nucleus. That work also attempted to bring together several other datasets to obtain a more global picture of the nucleus' properties. While less than perfect weather and the presence of coma presented real challenges, these investigators found that the effective radius at the time of the observation was 3.0+/-0.2 $\mathrm{km}$. To derive the mean effective radius (averaged over a rotation period), they had to make two important assumptions: first, that the observations referred to the maximum light in the rotational cycle (for which there was some observational evidence), and second, that the peak-to-valley range of $0.6+/-0.2$ magnitudes that is typical of most comets applied to 9P/Tempel 1's light curve as well. Given these assumptions, they found that the mean effective radius would be $2.6+/-0.2 \mathrm{~km}$. The geometric albedo was derived by first incorporating the Lamy et al. (2001) and Lowry et al. (1999) results with photometry from the database of Meech et al. (2004a). This let them derive a photometric characterization that was consistent with as many datasets as possible. They deduced an R-band absolute magnitude, $\mathrm{H}$, of about $14.5 \mathrm{mag}$. and a phase darkening parameter $\mathrm{G}$ of roughly -0.2 to obtain an R-band geometric albedo of $0.072+/-0.016$.

The surprisingly high value for the R-band geometric albedo from this study and the uncertainty in rotational phase led, in part, to a further investigation in the thermal infra-red (at several wavelengths between 8 and 22 microns) that utilized the newly launched Spitzer Space Telescope (Lisse et al., 2004a). While final results are not yet available, the full rotational light-curve variation was successfully covered and most of the uncertainty associated with rotational phase was eliminated. Preliminary results give a mean effective radius of $3.25 \pm 0.2 \mathrm{~km}$ and a V-band geometric albedo of $0.04 \pm 0.01$ (Lisse et al., 2004a). Working with the large database of ground-based R-band 
observations Hsieh and Meech (2004) have determined preliminary values for the R-band absolute magnitude $(14.441 \pm 0.067 \mathrm{mag})$ and together with the above result on mean effective radius we find a mean R-band geometric albedo of $0.05 \pm 0.01$. The run of absolute magnitude with solar phase angle, $\alpha$, is shown in Fig. 4. From this information we have been able to make a preliminary estimate for the phase law polynomial, $\Delta H(\alpha)$, at small phase angles where $H(1,1,0)=H(1,1, \alpha)+\Delta H(\alpha)$ and values for globally averaged Hapke parameters $\varpi_{0}, \mathrm{~h}, \mathrm{~S}(0)$, g, and $<\theta>$ (Helfenstein and Veverka, 1989). We find $\varpi_{0}=0.018, \mathrm{~h}=0.0135, \mathrm{~S}(0)=0.2, \mathrm{~g}=-0.525$, and $<\theta>=20 \mathrm{deg}$. (V-band), values that are similar to those found by Buratti et al. (2004) for 19P/Borrelly. The phase law polynomial (valid for $\alpha<15 \mathrm{deg}$ ) is: $\Delta \mathrm{H}(\alpha)=-0.0180955-0.2502604 \alpha+0.0306201 \alpha^{2}$ $-0.0021805 \alpha^{3}+0.0000798 \alpha^{4}-0.0000015 \alpha^{5} \mathrm{mag}$.

\subsection{THE SHAPE OF THE NUCLEUS.}

Appraisal of the nucleus' shape is intimately involved with the assessment of the spin state of the nucleus through its periodic brightness variations. If it can be assured that the lightcurve is free of the influence of cometary activity, as justified above, and that the variations are a reflection of the changing areal cross-section of the rotating nucleus, the peak-to-valley magnitude range gives an approximate value for the lower limit to the axial ratio (there is an implicit assumption that deviations from axial symmetry are not grotesquely large). If the direction of the rotational angular momentum vector (assumed fixed in space or at most slowly varying) is also known, then observations of the light curve range at different epochs can be translated into a rough estimate of the dimension of the nucleus. The magnitude range in Lamy et al. (2001) observations placed the 
earliest constraint, a lower limit to the axial ratio, $\mathrm{a} / \mathrm{b}=1.4$. An elongated nucleus was thereby established. Ground-based R-band data (Fig. 3), taken for the Deep Impact campaign during October, 2001, when the comet was at $4.15 \mathrm{AU}$ and phase angle of 14.1 deg, yielded an even greater magnitude range, 0.72 magnitudes, raising the lower limit of the nucleus axial ratio to 1.9. In Fig. 5 these data have been phased for the rotational period of 41.85 hrs (see below) and the slightly asymmetric, double-peaked, "saw-tooth" shape of the light curve is clearly seen. Our experience with modeling such curves suggests that the nucleus of 9P/Tempel 1 likely to be elongated and has considerable irregularities in its shape.

\subsection{THE ROTATION STATE.}

The set of ground-based photometric data that was primarily used in this work was taken beyond $4 \mathrm{AU}$ and consists of several groupings as shown in Fig. 2. One of these groups, B, is shown in detail in Fig. 3. Taken all together, these data show strong evidence of periodic variations with their mean (reduced to absolute magnitude) roughly independent of heliocentric distance. The analysis of these data is ongoing using the WindowClean algorithm and phase dispersion minimization based on simple harmonic wave model fits (for an up-to-date review of these techniques see Fernández et al., 2005), but by themselves they seem to be ambiguous about the actual spin period. We show a WindowClean periodogram for Group B in Fig.6. While a rotational period of $41.85 \pm$ $0.1 \mathrm{hr}$ provides a good accounting of the data for groups B, C and D, we also found that there is also a case for a period of, roughly, half that value $(\sim 22 \mathrm{hr})$ in other parts of the ground-based data. Published investigations provide little guidance with Weissman et al. 
(1999) suggesting a period in the range of 14 to $39 \mathrm{hr}$ and Lamy et al. (2001) a period in a range from 25 to $33 \mathrm{hr}$. To settle this issue we have obtained a new time series of nucleus magnitudes with the $H S T$ when the comet was at $3.53 \mathrm{AU}$. Although the data in Fig. 1 might suggest that there is still some possibility for a residual level of activity at this heliocentric distance, the HST data show no evidence of coma. The closely sampled data covers a sufficient length of time $(\sim 40 \mathrm{hr})$ to ensure a clear discrimination between the candidate rotational periods near 22 and 42 hours. Preliminary reductions (Fig. 7) yield a smooth, but asymmetric, double-peaked light curve with a period of near $40 \mathrm{hr}$ settling the issue of the rotation period and again suggesting an elongated and irregular shape. This result is reinforced by the newly acquired Spitzer Space Telescope data which also shows a double peaked light curve with a period $\sim 40 \mathrm{hrs.} \mathrm{The} \mathrm{Spitzer} \mathrm{and} H S T$ data, separated by $\sim 43$ days or roughly 25 cycles, can be combined to obtain a more accurate estimate of the period but, because of the large gap between the data sets the estimate is susceptible to aliasing. A preliminary study yields a preferred periodicity at $39.6 \pm 0.15$ hr, however, as seen in the phase plots of Fig. 8 , the period of $41.85 \pm 0.1 \mathrm{hr}$ obtained from ground-based data appears to give a better account of the light curve and we adopt this value as the rotational period of the nucleus. Eventually we expect that by combining all of the ground and space-based data sets, an effort that is ongoing, it should be possible to ultimately estimate the period with an accuracy approaching $\pm 10 \mathrm{sec}$, i.e., good enough to be able to predict the general orientation of the nucleus at encounter before launch of the spacecraft on January 8, 2004. 
In our studies so far we have found no evidence (e.g., a second periodicity noncommensurate with $41.85 \mathrm{hrs}$ ) for an excited spin state in this comet and we presume that the nucleus must spin in a state close or equal to that of principal axis rotation.

\subsection{THE DIRECTION OF THE ROTATION AXIS.}

To achieve a better estimate of the overall dimensions of the nucleus, knowledge of the spin pole direction is required. To achieve this, observations of the magnitude range of the nucleus lightcurve at three or more different viewing geometries are needed. In a preliminary study, we have used six independent light curves including the recently acquired HST and Spitzer Space Telescope observations. We used the "amplitude" method, which yields two distinct, but essentially photometrically equivalent, solutions for the pole (Magnusson et al. 1989). In Fig. 9 we show a series of intersecting small circles, each belonging to a single group of observations that traces out the locus of possible pole positions for an assumed axial ratio. The position on the sky where all of these small circles intersect at a common value is a solution for the direction of the rotation axis. Four such positions are identified which represent opposite ends of two photometrically equivalent pole positions. A good accounting of the various observations plus the best definition of the common point of intersection on the sky is obtained for an axial ratio $\mathrm{a} / \mathrm{b}=3.2 \pm 0.4$. This quantity is not, however well defined. The directions of the poles in the northern hemisphere are (RA, Dec, J2000) $=46 \pm 10,73 \pm 10$ deg (Pole 1) and $287 \pm 14,16.5 \pm 10 \mathrm{deg}$ (Pole 2) and, while it is not possible to be sure which is the true rotation axis, based on the scatter of intersection points in Fig. 9, pole 1 seems to give a better account of the data. The errors for the pole positions are based on the spread 
of the various intersection points on the sky and the underlying uncertainty of the location of the small circles as indicated in the figure. They are purposely given conservative values. The sense of spin remains unknown. By combining the axial ratio with the mean effective radius we find the overall dimensions of the nucleus to be approximately $14.2 \mathrm{x}$ $4.4 \times 4.4$. Using the pole directions found here we can calculate the aspect angle of the rotation axis as seen by the spacecraft on approach to encounter. As seen from the comet, the spacecraft is at RA, Dec $=20.4,-25.6$ on approach to encounter. The aspect angles for the two poles are therefore $82.2 \mathrm{deg}$ (Pole 1) and $69.7 \mathrm{deg}$ (Pole 2) - so the pole direction will be close to the plane of the sky. The predicted magnitude range for the light curve on approach is $\sim 1.2 \mathrm{mag}$, i.e., large. However, until the rotational period can be substantially improved, it is not yet possible to predict whether the impactor will have a broadside or end-on collision with the nucleus.

\subsection{ESTIMATED MASS, LOCAL GRAVITY, AND ESCAPE VELOCITY.}

Estimates of the value of these parameters may be important in understanding the details of the impact and the behavior of the ejecta. Using the nominal bulk density of 500 Kg.m-3 and approximating the shape with a prolate ellipsoid, we find a volume of $151 \pm$ $60 \mathrm{~km}^{3}$ and a mass of $\sim 7 \times 10^{13} \mathrm{~kg}$. The acceleration due to gravity at the long end of the object is $\sim 0.027 \mathrm{~cm} . \mathrm{s}^{-2}$ and at the waist $\sim 0.044 \mathrm{~cm} . \mathrm{s}^{-2}$. The corresponding escape velocities are 1.4 and $2.2 \mathrm{~m} . \mathrm{s}^{-1}$ respectively. Because of the long rotation period, the centrifugal and coriolis contributions are negligible. Because of the large uncertainty in the bulk density, the values quoted in this section are similarly uncertain. 


\subsection{COLOR AND SPECTRAL PROPERTIES.}

The V-R color of the bare nucleus of 9P/Tempel 1 in the Kron-Cousins photometric system is $0.56+/-0.02$ and was obtained when the comet was beyond $4 \mathrm{AU}$ from the sun from the data described in Meech et al. (2004a). Observations at smaller heliocentric distances when there was coma present, give colors (V-R) between $0.40<\mathrm{V}-\mathrm{R}<0.51$. The color of the active comet reflects a combination of composition and particle size, and is therefore more difficult to interpret. The solar color is $\mathrm{V}-\mathrm{R}=0.36$ (Livingstone, 2000; Fernie, 1973), and the average nucleus color is about V-R $=0.42 \pm 0.02$ (Meech et al, 2004b) for a sample of 17 comets. Thus, cometary nuclei are in general red with respect to the sun (although there is a large range colors, with some being bluer) and among comet nuclei, 9P/Tempel 1 is quite red. We know there is a trend of reddening as a function of increasing heliocentric distance in the asteroid belt, and that Transneptunian Objects (TNOs) and Centaurs can be extremely red, and have a large diversity of surface colors, possibly due to the presence of organic material and ion irradiation and other weathering processes. Also, cometary activity is likely to alter surface composition by redistributing dust or creating lag deposits on localized areas of the surface, or by removing weathered (possibly reddened) materials. As discussed in Meech et al. (2004b), comet colors on average are shifted toward bluer colors with respect to Kuiper Belt Objects (KBOs) and Centaur colors, which have average V-R between 0.54 - 0.63 (Hainaut and Delsanti, 2002), however, the range or distribution of colors is as broad as those of the more distant objects.

As no visible or near infrared spectrum of the comet's nucleus is available, the spectral properties of the nucleus surface are unknown. Our expectations to explore the 
near-IR spectrum $(1-5 \mu)$ of 9P/Tempel 1 are discussed in a companion paper (Sunshine et al., 2004). Abell et al. (2003) obtained a high quality spectrum of the surface of comet C/2001 OG108 (LONEOS) and, aside from Deep Space 1's partially saturated spectrum of 19P/Borrelly (Soderblom et al., 2004b), is perhaps the best representation of the nearinfrared reflectance spectrum of a comet nucleus available. The spectrum is featureless and mimics that of a P-type asteroid (slightly red, largely featureless, and is distinguished by a low geometric albedo in the optical range) quite closely. We anticipate that the nearinfrared spectrum of 9P/Tempel 1 will be similar. A reasonable explanation for the spectrum is thought to be the dominant role of low albedo, highly absorbing, mix of organic material in the reflectivity of the surface material. Therefore most spectral signatures of silicates and ices that might be expected to constitute the primary materials in the crust can expected to be only weakly evident. The Deep Space $1(1.3-2.6 \mu)$ spectrum of 19P/Borrelly shows a spectral reflectance that increases with increasing wavelength after removal of an appreciable thermal component beyond 1.8 microns. There is also an unidentified absorption band near 2.4 microns which may possibly be present in the spectrum of 9P/Tempel 1.

A thermal spectrum has been obtained with the Spitzer Space Telescope but its calibration is at present uncertain. 


\section{Anticipated Properties of the Surface and Subsurface}

\subsection{ACTIVE REGIONS.}

An important issue for the Deep Impact mission is the identification of possible correlations between localized areas (active areas) and jet activity in the inner coma. As an example, the results of such a study for comet $81 \mathrm{P} /$ Wild 2 have recently been reported by Sekanina et al. (2004). They find that cometary activity can flourish perhaps even in unilluminated regions - already an important result that needs confirmation. In the case of 9P/Tempel 1 there are many pieces of evidence suggesting that only a small fraction of the total surface area can actively release dust and gas. Early estimates of the active area required to produce the observed $\mathrm{OH}$ release yielded an active area of roughly $4 \mathrm{~km}^{2}$ (A'Hearn et al. 1995), indicating that only $4 \%$ of the total surface area as deduced by Lamy et al. (2001), was active (according to Groussin [personal communication], this result contains a numerical error of a factor of two and the value should be increased to 8\%). Lisse et al, (2004a), using a mean effective radius of $3.25 \mathrm{~km}$, derived from a thermal model, an active fraction of $9 \pm 2 \%$ at the time of perihelion i.e., an area of roughly $17 \mathrm{~km}^{2}$. Both of these estimates indicate a relatively low level of activity compared to comet nuclei like 46P/Wirtanen ( $\sim 100 \%$ at perihelion, Groussin and Lamy, 2003), 22P/Kopff (> 35\%, Lamy et al., 2002) or 103P/Hartley $2(\sim 100 \%$ at perihelion, Groussin et al., 2004). The shape of the comet's heliocentric light curve may also imply discrete active areas because it peaks about three months prior to perihelion and the activity has dropped by a factor of two by the time of perihelion Light curves by C.H. Morris can be found on the web at http://encke.jpl.nasa.gov/comets_short/9P.html). Such behavior may require a heterogeneous outer layer for the nucleus and could be interpreted 
as a seasonal effect due to a small number of discrete active areas. In Fig. 10 we show direct evidence of discrete active areas: drawings of the inner regions of the comet's coma in April, 1983, and April, 1994 show jet structures that have generally a similar morphology at the two different times. Since the orbital period of the comet is almost exactly 5.5 years and assuming the active areas are constant from one apparition to the next, the geometry of Earth-based observations will repeat every 11 years. The jet structures may therefore be expected to repeat, for a given rotational phase. We expect that, at encounter in July 2005, we will observe several discrete active areas on the nuclear surface, much as did the Stardust mission during its recent encounter with comet 81P/Wild 2 (Brownlee et al., 2004; Sekanina et al., 2004).

\subsection{SURFACE MORPHOLOGY.}

Images obtained during the recent encounters of Stardust with 81P/Wild 2 and Deep Space 1 with 19P/Borrelly provide the most detailed information that we have on the surface morphology on cometary nuclei. These images with resolutions as high as 14 m/pixel (Brownlee et al. 2004; Soderblom et al., 2004a) show two apparently distinct types of surface topography that are possibly related to the sublimation history of the two objects (Brownlee et al. 2004). Nevertheless, both exhibit rugged topography at the scale of $\sim 100 \mathrm{~m}$. In the case of $81 \mathrm{P} /$ Wild 2 we find large, quasi-circular, features, some of which may have an impact origin, steep cliffs, spires and evidence for overhanging walls; in the case of 19P/Borrelly, the scene is more subdued (possibly partly due to the lower resolution at 19P/Borrelly) but stereoscopic evidence is clearly there for mesa-like structures and large scale fracture systems separating distinct elements of the comet's 
nucleus. At smaller scales pits and ridges are seen. There is also stereo-photometric evidence for appreciable variations in surface roughness from one place to another (Kirk et al., 2004). In a companion paper (Thomas et al., 2004) examine the question of what geological features might be expected in the case of 9P/Tempel 1 and we refer detailed discussion to that paper. Suffice it to say that the surface geology is expected to be dominated by processes associated with the ablation of volatile materials and the formation of lag deposits. The characteristics of these features are expected to be shaped by inhomogeneities in the local mix of materials, variations in slope, shadowing etc. Craters, particularly small craters, are not expected to dominate the landscape since erosion associated with ablation is expected to be rapid process. The increase in surface resolution by a factor of four or five, to $\sim 3 \mathrm{~m} / \mathrm{pxl}$ and possibly even higher, plus the acquisition of very high spatial resolution $(\sim 10 \mathrm{~m} / \mathrm{pxl})$ color maps should yield new discoveries about the surface morphology. More significantly, the combination of resolution, stereography, and color that is expected to be achieved by Deep Impact imaging should advance the exploration of cometary surfaces well beyond simple photogeological reconnaissance and possibly reach a level where the nature of ongoing geological processes can be understood.

\subsection{COMPOSITION.}

The surface composition and the bulk composition of the comet are expected to be different as a result of volatile depletion in the surface layers (see below). The bulk composition for any comet has yet to be measured, but theoretical ideas abound. As a working hypothesis, we chose to base our estimate on the analysis of Huebner (2003) that 
yields a roughly equal split (based on mass fraction) between volatile ices, carbonaceous material, and silicates. While accurate measures of various species may be available for observables in the coma (Lisse et al., 2004b), model studies show that the extrapolation of these numbers into the source regions in the interior of the nucleus is fraught with uncertainty (Huebner and Benkhoff, 1999).

So far direct mass spectrometric data sets are available for two comets: The first is for 1P/Halley, obtained in 1986, from the PUMA $1 \& 2$ and PIA instruments on the VEGA 1\&2 and Giotto missions (Kissel et al.,1986a, 1986b). More recently, Jan 4, 2004, the CIDA instrument on the Stardust mission (cf. Kissel et al., 2003) provided similar data for 81P/Wild 2 (Kissel et al.,2004a). While the data for 1P/Halley were obtained at a flyby speed of over $67 \mathrm{~km} / \mathrm{s}$, the relative speed at $81 \mathrm{P} /$ Wild 2 was only $6.1 \mathrm{~km} / \mathrm{s}$. At the higher speed mainly atomic positive ions were measured, which lessened the chance to be able to characterize the organic material (Kissel and Krueger, 1987) using molecular ions. At the lower speed the molecular ions dominate, while mineral ions are almost undetectable (Kissel et al., 2004). The two sets of measurements were complemented by measurements of the interstellar dust during cruise (Krueger et al., 2004). While the 1P/Halley data suggested the presence of a chemical class of PAHs with $\mathrm{O}$ and $\mathrm{N}$ as hetero-atoms, the interstellar sample contained a specific class of PQQ (Pyrroloquinoline quinine: 4,5-dihydro-4,5-dioxo-1 $H$-pyrrolo-[2,3-f]quinoline-2,7,9-tricarboxylic acid) like molecules (Krueger et al., 2004). In addition, a relative enhancement of $\mathrm{N}$ bearing molecules was found for $81 \mathrm{P} /$ Wild 2 . These could be derived from the interstellar molecules by the elimination of $\mathrm{H}_{2} \mathrm{O}$ and $\mathrm{CO}$. Not only are the PQQ-like molecules likely to release $\mathrm{H}_{2} \mathrm{O}$ and $\mathrm{CO}$ upon initial heating, but they can also store substantial amounts of 
latent energy from bombardment with cosmic rays. Oberc $(1993,1996)$ argues, on the basis of measurements with VEGA 2 and the global properties of the organic material reported by Krueger et al. (1991) that the latent energy could be as high as $16 \mathrm{~kJ} / \mathrm{mol}$. Due to the latent energy potential, possibly buried in the cometary organic material, we should perhaps not be surprised to find the impact to liberate a substantial amount of material by this energy source and not just from the impact energy alone.

\subsection{MATERIAL STRENGTH.}

The strength of the material in the nucleus is very uncertain, no matter whether we consider tensile strength, shear strength, or compressive strength. The tidal disruption of comet D/Shoemaker-Levy 9 (S-L 9) by Jupiter sets a rough upper limit to the tensile strength of about 1000 dyn. $\mathrm{cm}^{-2}$ for spatial scales near $1 \mathrm{~km}$ and allows for strengths approaching zero (e.g., Asphaug and Benz, 1996). The apparent success of a rubble-pile interior model in predicting the distribution of the fragments of S-L 9, suggests that the tensile strength in 9P/Tempel 1 may be similarly low at spatial scales much less than 1 $\mathrm{km}$. Nevertheless, the existence of overhangs of $100-150 \mathrm{~m}$ in the Stardust images of 81P/Wild 2 (Brownlee et al. 2004) requires that the strength on these shorter scales not be zero. The gravity is probably so weak on these objects that a tensile strength of $\sim 100$ dyn. $\mathrm{cm}^{-2}$ on scales of 10 to $100 \mathrm{~m}$ could be sufficient to support such structures. Because gravity acting on a coherent overhang will produce a torque - stretching the upper surface of the overhang from the wall while compressing the lower surface against the wall - this estimate will represent some combination of compressive and tensile strength. Davidsson (2001) estimated the lower limits of comet tensile strengths to escape 
rotational breakup for a selection of comets with a range of possible densities at between 10 and 530 dyne. $\mathrm{cm}^{-2}$. A further and comparative study has been performed for comets and asteroids to estimate the tensile strengths and bulk densities by Toth (2001) and it was found that the vast majority of observed comets are stable against rotational breakup because they are in the stable domain in the rotational period - effective radius diagram. Finally, the interstellar aggregate dust model (Greenberg et al., 1995), predicts a bulk material strength at $\sim 0.88 \rho$ (SI units), where $\rho$ is the mean bulk density of the nucleus. This leads to an estimate of 440 dyne. $\mathrm{cm}^{-2}$. For Deep Impact we adopt a value of $500 \pm$ 400 dyne. $\mathrm{cm}^{-2}$ for the bulk strength of the material on the nucleus of 9P/Tempel 1.

Near the surface of the nucleus, particularly at small linear scales, the material strength may be very much higher as a result of processes such as radiation sintering (Smoluchowski, 1989) as the material structure has evolved into a crust under the influence of solar insolation (Kührt and Keller, 1994). Their estimates of the local strength (i.e at small scales) are as high as $10^{7}$ dynes.cm ${ }^{-2}$. As suggested by Kührt and Keller (1994), the build-up of a strong crust, perhaps a few meters thick, may be essential to understanding the observed existence of widespread regions of surface inactivity. Since the Deep Impact impactor spacecraft is like to strike the surface in such an inactive area, these concepts may become essential elements in understanding the nature of the crater forming process that is observed.

\subsection{SUBSURFACE AND INTERIOR STRUCTURE.}

Comet 9P/Tempel 1 appears to be a typical Jupiter family comet. Its orbital history suggests that it has been in a relatively stable configuration with non-gravitational 
parameters (and by implication spin state and outgassing rate) that are "unusually constant from one apparition to the next" (Yeomans et al. 2000). It follows an orbit that, at least for the last 400 yrs (the time for which orbital integrations are considered reliable), is close to a 2:1 resonance with Jupiter. The orbit oscillates with extended periods where the perihelion distances is either near 1.5 or $2 \mathrm{AU}$ (Yeomans et al., 2004). This situation should have provided a reasonably stable environment for aging of the surface and for subsurface differentiation of volatiles. At formation, a comet nucleus is expected to include many different volatile species. If cometary water ice is crystalline, these volatiles will be frozen out as separate phases; otherwise, volatiles maybe trapped in the amorphous ice. Clathrate hydrates are now considered a less likely possibility as their formation in impure ice at low temperatures is unlikely (Jenniskins and Blake, 1996). The nature of cometary ice is still uncertain; the Deep Impact mission is bound to shed light on this puzzle. As the heat absorbed at the surface penetrates the porous nucleus, ices will evaporate from the pore walls and the gas will flow, in part, to the surface and out of the nucleus, and in part, to the colder interior where it will refreeze. Since evaporation rates are strongly temperature dependent and vary widely among gas species, several distinct evaporation fronts are expected to form and also, several separate layers of refrozen gases, although the ice mixture may have been homogeneous initially. If the water ice is amorphous, the trapped gas will escape when the ice crystallizes. In this case, all species will escape together and, generally, at higher temperatures than those typical of evaporation. However, once they are released from the ice, these gases will behave similarly to gases that evaporate from the pore walls. Again, a chemically differentiated layered structure will emerge (Prialnik et al. 2004). 
Thus models show that a layered structure will occur regardless of the nature of the water ice, starting at a depth of $10 \mathrm{~cm}$ to a few tens of meters (Strazzula and Johnson 1991, Prialnik and Mekler 1991, Benkhoff and Huebner 1995, Klinger 1996). A more accurate picture is difficult to predict, since it depends on physical properties, as well as thermal history. In Jupiter family comets the water is expected to be crystalline above this differentiated layer, even if it was amorphous at formation. The outer layer of crystalline ice - essentially, the orbital skin depth of the nucleus - will be depleted of more volatile ices. Models also show that at an exposed (active) area, the porosity is bound to be high near the surface; in contrast, a dust-covered area may have a crust of dense (low-porosity) ice just beneath it. The dust layer covering inactive regions may be as thin as a few $\mathrm{cm}$ (Rickman et al. 1990, Prialnik and Mekler 1991, Coradini et al. 1997), which is sufficient for insulating the ice-rich material beneath it and reducing sublimation by a large factor.

The interior structure on a global scale is yet another mystery. Models range from a fluffy mix of largely interstellar material to accumulations of cometesimals rather like the asteroidal "rubble pile' model (for an up-to-date review of our understanding of cometary interiors see the reviews of Donn, 1991, and Weissman et al., 2004). The only quantitative information that might bear on its interior are the number of pieces that were observed as a result of the tidal break-up of comet D/Shoemaker-Levy-9 near Jupiter (Asphaug and Benz, 1996) and the expectation for near homogeneity of its internal mass distribution based on results for 1P/Halley. In this case the internal mass distribution of the nucleus is constrained by a combination of observations of its observed shape and the two ratios of principal moment of inertia that follow from the observed excited spin state (Belton et al., 1991). At the surface, as noted above, the steep walls, spires, and 
overhangs seen on $81 \mathrm{P} /$ Wild 2 (Brownlee et al. 2004) provide qualitative evidence for an increase in strength (and perhaps sealing in of volatiles) in the upper layers of the nucleus that may have occurred as a result of a protracted period of compositional and structural evolution under the influence of sunlight (Kührt and Keller, 1994).

\section{Conclusions}

Many diverse lines of evidence, including the results of missions to 1P/Halley, 19P/Borrelly, and 81P/Wild 2, allow us to formulate a reasonably consistent picture of conditions on 9P/Tempel 1. Also, major recent advances in our observational characterization of the nucleus of 9P/Tempel 1 have occurred as a result of a massive and highly successful Earth-based observational campaign organized by the Deep Impact science team (Meech et al., 2004a; Lisse et al., 2004a). Preliminary estimates, partially based on this database, allow us to specify a set of working parameters (Table I), which will be used by the team in planning for the encounter and impact. They will also provide a guide in the initial data analysis phase. The value of many of these quantities can be expected to be modified, or updated, as a result of this ongoing campaign, the ongoing data analysis, and the results of further observations taken from the Deep Impact spacecraft and from the vicinity of Earth around the time of impact. For now we must be satisfied with the set of working parameters in this paper. 


\section{References}

Abell, P. A., Fernandez, Y. R., Pravec, P., French, L. M., Farnham, T. L., Gaffey, M. J., Hardersen, P. S., Kusnirak, P., Sarounova, L., and Sheppard, S. S. : $2003,34^{\text {th }}$ Lunar \& Planet. Science Conf. (Abstract)

A’Hearn, M.F., Belton, M.J.S., and Delamere, A.: 2004, Submitted for publication in Space Science Reviews

A’Hearn, M.F., Millis, R.L., Schleicher, D.G., Osip, D.J., and Birch, P.V.: 1995, Icarus, 118, 223-270.

Asphaug, E. and Benz, W.: 1996, Icarus, 121, 225-248.

Belton, M.J.S., Samarasinha, N.H., Fernandez, Y.R., and Meech, K.J.: 2005. Accepted for publication in Icarus, Nov. 2004.

Belton, M.J.S., Julian, W.H., Anderson, A.J., and Mueller, B.E.A.: 1991, Icarus, 93, 183-193.

Benkhoff, J., and Huebner, W.: 1995, Icarus, 114, 348 - 354.

Brownlee, D. E., Horz, F., Newburn, R. L., Zolensky, M., Duxbury, T. C., Sandford, S., Sekanina, Z., Tsou, P., Hanner, M. S., Clark, B. C., Green, S. F., and Kissel, J.: 2004, Science, 304, 1764 - 1769.

Buratti, B.J., Hicks, M.D., Soderblom, L.A., Britt, D., Oberst, J., and Hillier, J.K.: 2004. Icarus 167, 16 29.

Coradini, A., Cappacioni F., Capria, M. T., De Sanctis, M. C., Espinasse, S., Orosei, R., and Salomone M. 1997, Icarus 129, 337-347.

Davidsson, B.J., 2001, Icarus 149, 375-383.

Donn, B.: 1991, In Newburn, R.L., Neugebauer, M., and Rahe, J., (Eds.) Comets in the Post-Halley Era. Kluwer Academic Publishers, Dordrecht.

Fernandez, Y.R., Lowry, S.C., Weissman, P.R., Mueller, B.E.A., Belton, M.J.S., and Meech. K.J.: 2005, Accepted for publication in Icarus, Nov. 2004.

Fernández, Y.R., Meech, K.J., Lisse, C.M., A’Hearn, M.F., Pittichová, J., and Belton, M.J.S.: 2003, Icarus, $164,481-491$.

Fernie, J.D,.:1973. Pub. Astron. Soc. Pacific 95, 782.

Greenberg, J.M., Mizutani, H., Yamamoto, T.: 1995, Astron. Astrophys. 295, L35-L38.

Groussin, O., Lamy, P., Jorda, L., and Toth, I.: 2004. Astron. \& Astrophys., 419, 375 - 383. 
Groussin, O. and Lamy, P.: 2003. Astron. \& Astrophys., 412, 879 - 891.

Hampton, D., Wellnitz, D.: 2004. Submitted for publication in Space Science Reviews

Hainaut, O.R., and Delsanti, A.C.:2002. Astron. \& Astrophys. 389, 641 - 664.

Hapke, B.: 1984. Icarus 59, $41-59$.

Helfenstein, P., and Veverka, J.: 1989. In Binzel, R.P., Gehrels, T., and Mathews, M.S. (Eds.) Asteroids II. University of Arizona Press, Tucson

Hsieh, H.H., and Meech, K.J.: 2004, personal communication.

Huebner, W.F.:2003, In Cometary Science after Hale-Bopp. I. (Boehnardt, H., et al., Eds.). Kluwer Academic Publications, Dordrecht.

Huebner, W.F., and Benkhoff, J.: 1999, Space Science Rev. 90, 117-130.

Jenniskins, P., and Blake, D.F.: 1996. Astrophys. J, 473, $1104-1113$.

Jewitt, D.C., and Meech, K.J.: 1988, Astrophys. J., 328, 974-986.

Klaasen, K.P., Carcich, B., Carcich,G. and Grayzeck, E.:2004. Submitted for publication in Space Science Reviews.

Klinger, J.:1996, In H. Rickman et al (eds). Proc. First Comet Nucleus Surface Properties Workshop. Preprint.

Kirk, R.L., Howington-Krauss, E., Soderblom, L.A., Giese, B., and Oberst, J.: 2004, Icarus 167, 54 - 69.

Kissel, J., Krueger, F.R., Silén, J., and Clark, B.C.: 2004, Science 304, 1774-1776.

Kissel, J.; Glasmachers, A.; Grün, E.; Henkel, H.; Höfner, H.; Haerendel, G.; von Hoerner, H.; Hornung, K.; Jessberger, E. K.; Krueger, F. R.; Möhlmann, D.; Greenberg, J. M.; Langevin, Y.; Silén, J.; Brownlee, D.; Clark, B. C.; Hanner, M. S.; Hoerz, F.; Sandford, S.; Sekanina, Z.; Tsou, P.; Utterback, N. G.; Zolensky, M. E.; Heiss, C. : 2003, J.Geophys. Res.108, 401.

Kissel, J. and Krueger, F.R.: 1987, Nature, 326, 755-760.

Kissel, J., Brownlee, D.E, Büchler, K., Clark, B.C., Fechtig, H., Grün, E., Hornung, K., Igenbergs, E.B., Jessberger, E.K., Krueger, F.R., Kuczera, H., McDonnell, J.A.M., Morfill, G.E.,Rahe, J., Schem, G.H., Sekanina, Z., Utterback, N.G., Völk, H.J., and Zook, H.: 1986a, Nature,321, 336-337.

Kissel, J., Sagdeev, R.Z., Bertaux, J.L., Angarov, V.N., Audouze, J., Blamont, J.E., Büchler, K., von Hoerner, H., Inogamov, N.A., Khromov, V.N., Knabe, W., Krueger, F.R., Langevin, Y., Levasseur- 
Regourd, A.C., Managadze, G.G.,Podkolzin, S.N., Sharipo, V.D., Tabaldyev, S.R., and Zubkov, B.V.: 1986b, Nature, 321, 280- 282.

Krueger F.R., Werther W., Kissel J., and Schmid E.J.:2004, Rapid Commun. Mass Spectrom. 18, 103-111.

Krueger, F.R., Korth, A., and Kissel, J.: 1991 Space Science Reviews 56, 167-175.

Kührt, E., and Keller, H.U.:1994, Icarus 109, 121-132.

Lamy, P. Toth, I., Fernández, Y.R., and Weaver, H.A.: 2004, In M.C. Festou et al. (eds). Comets II. University of Arizona Press. Tucson

Lamy, P. Toth, I., Jorda, L., Groussin, O., A'Hearn, M.F., and Weaver, H.A.: 2002. Icarus, 156, 442 455.

Lamy, P.L., Toth, I., A’Hearn, M.F., Weaver, H.A., and Weissman, P.: 2001, Icarus, 154, 337-344.

Lisse, C., A’Hearn, M.F., Groussin, O., Fernández, Y.R., Belton, M.J.S., Van Cleve, J.E., Charmandaris, V., Meech, K.J., and McGleam, C.: 2004a. To be submitted to Astrophys. J. Lett.

Lisse, C., A’Hearn, M.F., Farnham, T., Groussin, O., Meech, K.J., and Fink, U., and Schleicher, D.G.:2004, Submitted for publication in Space Science Reviews.

Livingstone, W.C.: 2000. In Cox, A.N. (Ed.), Allen's Astrophysical Quanitities. Springer, New York. Lowry, S.C., and Weissman, P.R.: 2003. Icarus 164, $492-503$.

Lowry, S.C., Fitzsimmons, A., Cartwright, I.M., and Williams, I.P.:1999, Astron. Astrophys, 349, 649-659.

Magnusson, P., Barucci, M.A., Drummond, J.D., Lumme, K., Ostro, S.J., Surdej, J., Taylor, R.C., and Zappalà, V.: 1989. In Binzel, R.P., Gehrels, T., and Mathews, M.S. (eds). Asteriods II. University of Arizona Press, Tucson

Meech, K.J., et al.: 2004a, Submitted for publication in Space Science Reviews.

Meech, K. J., Hainaut, O.R., and Marsden, B.G.:2004b, Icarus 170, 463 - 491.

Meech, K.J., Fernandez, Y., and Pittichova, J. 2001. Bull. Amer. Astron. Soc. 33, 1075.

Oberc, P.: 1993, Planet. Space Sci. 41, 609-617.

Oberc, P.: 1996, Icarus 124, 195-208.

Peale, S.J.:1989. Icarus 82, $36-49$.

Prialnik, D. Benkhoff, J., and Podolak, M. 2004, in Festou, M. (Ed.) Comets II, Univ. of Arizona Press, Tucson. 
Prialnik, D., and Mekler, Y.:1991, Astrophys. J., 366, 318 - 323.

Rickman H., Fernandez, J. A., and Gustafson, B. A. S. 1990, Astron. Astrophys. 237, 524-535.

Rickman, H.: 1989. Adv. Space Res. 9, 59 - 71.

Rickman, H. 1986. ESA SP-249, 195 - 205.

Sagdeev, R.Z., Elyasberg, P.E., and Moroz, V.I.: 1988. Nature 331, 240 - 242.

Samarasinha, N.H., and Belton, M.J.S.: 1995, Icarus, 116, 340-358.

Sekanina, Z., brownlee, D.E., Economou, T., Tuzzolino, A.J., and Green, S.F.: 2004, Science, 304, 1769 1774.

Skorov, Y.V., and Rickman, H.: 1999. Planet. Space Sci. 47, 935 - 949.

Smoluchowski, R.: 1989, Astron. J. 97, 241-245.

Soderblom, L.A., Boice, D.C., Britt, D.T., Brown, R.H., Buratti, B.J., Kirk, R.L., Lee, M., Nelson, R.M., Oberst, J., Sandel, B.R., Stern, S.A., Thomas, N., and Yelle, R.V.: 2004a, Icarus, 167, 4-15.

Soderblom, L.A., Britt, D.T., Brown, R.H., Buratti, B.J., Kirk, R.L., Owen, T.C., and Yelle, R.V.: 2004b, Icarus, $\mathbf{1 6 7}, 100-112$.

Strazzula, G., and Johnson, R.E.: 1991, In Newburn, R.L., Neugebauer, M., and Rahe, J.(eds.) Comets in the Post-Halley Era. Kluwer Academic Publishers, Dordrecht.

Sunshine, J., A’Hearn, M.F., Groussin, O., McFadden, L-A., Klaasen, K.P., Schultz, P.H., and Lisse, C.M.: 2004. Submitted for publication in Space Science Reviews.

Thomas, P.C, Veverka, J., A’Hearn, M.F., McFadden, L., and Belton, M.J.S.: 2004. Submitted for publication in Space science Reviews.

Toth, I.: 2001, In Asteroids 2001: From Piazzi to the 3rd Millennium, Abstr. No. VIII. 11, p. 313. Palermo, Italy.

Weissman, P.R., Doressoundiram, A., Hicks, M., Chamberlin, A., and Hergenrother, C.: 1999, Bull. Am. Astron. Soc., 31, 1121.

Weissman, P.R., Asphaug, E., and Lowry, S.C.:2004. In Festou, M. (Ed.) Comets II, Univ. of Arizona Press, Tucson.

Yeomans, D. K., Giorgini, J., and Chesley, S.: 2004, Submitted for publication in Space Science Reviews. 
Yeomans, D.K., Chamberlin, A.B., Chodas, P.W., Keesey, M.S., and Wimberly, R.N.: 2000. Jet Propulsion Laboratory Interoffice Memorandum. 
TABLE I: Working Properties for the Nucleus of Comet 9P/Tempel 1

\begin{tabular}{|c|c|c|c|}
\hline Property & Nominal Values & Reference & Notes \\
\hline Mean density $\left(\mathrm{kg} / \mathrm{m}^{3}\right)$ & $500 \pm 400$ & See text & No direct measurements available \\
\hline Mean mass (kg) & $\sim 7 \times 10^{13}$ & See text & Range: $0.05-1.3 \times 10^{14} \mathrm{~kg}$ \\
\hline Mean radius $(\mathrm{km})$ & $3.25 \pm 0.2$ & Lisse et al (2004) & Spitzer data \\
\hline Shape & Elongated, irregular & This work & Non-sinusoidal light curve \\
\hline Axial ratio $(\mathrm{a} / \mathrm{b})$ & $3.2 \pm 0.4$ & This work & From pole solution \\
\hline Dimensions (km) & $\mathrm{a}=7.2 \pm 0.9 ; \mathrm{b}=\mathrm{c}=2.2 \pm 0.3$ & This work & $\begin{array}{c}\text { Uses } \mathrm{a} / \mathrm{b}=3.2 \pm 0.4 \text { and the } \\
\text { tabulated mean radius }\end{array}$ \\
\hline Estimated volume $\left(\mathrm{km}^{3}\right)$ & $151 \pm 60$ & This work & Derived from shape \\
\hline Estimated Surface area $\left(\mathrm{km}^{2}\right)$ & $143 \pm 18$ & This work & Derived from shape \\
\hline Spin state & Principal axis rotation & This work & Preliminary result \\
\hline Rotation period & $41.85 \pm 0.1 \mathrm{hr}$ & This work & Preliminary result \\
\hline $\begin{array}{l}\text { Northern Pole direction } \\
\text { (RA [deg], Dec [deg]. Epoch J2000) }\end{array}$ & $\begin{array}{l}\text { Pole 1: }(46 \pm 10,73 \pm 10) \\
\text { Pole 2: }(287 \pm 14,16.5 \pm 10)\end{array}$ & This work & $\begin{array}{l}\text { Photometrically, but not } \\
\text { geometrically, equivalent poles }\end{array}$ \\
\hline Surface gravity $\left(\mathrm{cm} \cdot \mathrm{s}^{-2}\right)$ & $\begin{array}{c}\text { Pole: } \sim 0.04 \\
\text { Long end } \sim 0.027\end{array}$ & This work & $\begin{array}{c}\text { Density }=500 \mathrm{~kg} \cdot \mathrm{m}^{-3} ; \text { Rotation has } \\
\text { little effect }\end{array}$ \\
\hline Escape velocity $\left(\mathrm{m} \cdot \mathrm{s}^{-1}\right)$ & $\begin{array}{c}\text { Pole: } \sim 2.2 \\
\text { Long end: } \sim 1.4\end{array}$ & This work & $\begin{array}{c}\text { Density }=500 \mathrm{~kg} \cdot \mathrm{m}^{-3} ; \text { Rotation has } \\
\text { little effect }\end{array}$ \\
\hline Mean R-band Absolute Magnitude & $14.441 \pm 0.067$ & $\begin{array}{l}\text { Hsieh and Meech } \\
\quad(2004)\end{array}$ & Preliminary result. \\
\hline $\begin{array}{l}\text { Estimated mean phase law: } \Delta H(\alpha) \\
\text { mag. }(\alpha=\text { phase angle in degrees })\end{array}$ & \multicolumn{2}{|c|}{$\begin{array}{c}-0.0180955-0.2502604 \alpha+0.03062 \alpha^{2}-0.0021805 \alpha^{3}+ \\
0.0000798 \alpha^{4}-0.0000015 \alpha^{5}\end{array}$} & $\begin{array}{l}\text { Preliminary result see text. } \\
\text { Good for } \alpha<15 \text { deg }\end{array}$ \\
\hline Estimated V-band Hapke parameters & \multicolumn{2}{|c|}{$\begin{array}{c}\varpi_{0}=0.018, \mathrm{~h}=0.0135, \mathrm{~S}(0)=0.2 \\
\mathrm{~g}=-0.525,<\theta>=20 \mathrm{deg}\end{array}$} & Preliminary result. This work. \\
\hline Geometric Albedo (V, R-bands) & $0.04 \pm 0.01,0.05 \pm 0.01$ & $\begin{array}{l}\text { (V-band) Lisse et al. } \\
\text { (2004a) and (R-band) } \\
\text { this work }\end{array}$ & $\begin{array}{l}\text { Based on tabulated Abs. Mag. And } \\
\text { mean radius }\end{array}$ \\
\hline Color (V-R) [Kron - Cousins system] & $0.56 \pm 0.02$ & $\begin{array}{l}\text { Hsieh and Meech } \\
(2004)\end{array}$ & Preliminary result \\
\hline Spectral Reflectance & $\sim$ P-type asteroid & Abell et al. (2003) & $\begin{array}{l}\text { Inferred from spectrum of other } \\
\text { comets }\end{array}$ \\
\hline Composition & $\begin{array}{c}\text { Silicate dust }(\sim 33 \%) \\
\text { Organics }(33 \%) ; \text { Ices }(33 \%) \\
\end{array}$ & See text & Huebner (2003) \\
\hline Gas Production Rate $\left(\mathrm{Q}_{\mathrm{OH}}\right)$ & $1.7-2.15 \times 10^{28} \mathrm{~mol}_{\mathrm{s}} \mathrm{s}^{-1}$ & A’Hearn et al. (1995) & At perihelion \\
\hline Dust Production Rate (Af $\rho$ ) & $293 \mathrm{~cm}$ & $\begin{array}{l}\text { A'Hearn et al. (1995) } \\
\text { (at perihelion) }\end{array}$ & $\begin{array}{l}\text { Marked asymmetry about } \\
\text { perihelion }\end{array}$ \\
\hline Active fraction of surface $(\%)$ & $9 \pm 2$ & $\begin{array}{l}\text { A'Hearn et al (1995) } \\
\text { Lisse et al. (2004a) }\end{array}$ & $\begin{array}{l}\text { From } \mathrm{OH} \text { production rate } \\
\text { and estimated surface area }\end{array}$ \\
\hline Thermal inertia & $0-100 \mathrm{~J} / \mathrm{K} / \mathrm{m}^{2} / \mathrm{s}^{1 / 2}$ & Lisse et al. (2004a) & Preliminary result \\
\hline Surface Morphology & \multicolumn{2}{|c|}{$\begin{array}{l}\text { Rugged at the scale of } 100 \mathrm{~m} \text { but physically weak. } \\
\text { Possibility of impact craters }\end{array}$} & $\begin{array}{c}\text { By analogy to } 19 \mathrm{P} / \text { Borrelly and } \\
\text { 81P/Wild } 2\end{array}$ \\
\hline Subsurface & \multicolumn{2}{|c|}{ Volatile differentiation to several 10 's of meters } & $\begin{array}{l}\text { Working hypothesis based on } \\
\text { theoretical models }\end{array}$ \\
\hline Interior mass distribution & Effectively homogeneous & Belton et al. (1991) & By analogy to $1 \mathrm{P} /$ Halley \\
\hline
\end{tabular}




\begin{tabular}{|l|c|c|c|}
\hline Interior structure & $\begin{array}{c}\text { Weak accumulation of } \\
\text { cometesimals }\end{array}$ & $\begin{array}{c}\text { Asphaug and Benz } \\
\text { (1996) }\end{array}$ & Analogy to D/Shoemaker-Levy 9 \\
\hline Large scale tensile strength & $500 \pm 400$ dynes.cm ${ }^{-2}$ & See text & See text \\
\hline Crustal (small scale) strength & Up to $10^{7}$ dynes.cm ${ }^{-2}$ & Smoluchowski (1989) & $\begin{array}{c}\text { Crustal development (Kührt and } \\
\text { Keller,1994) }\end{array}$ \\
\hline Insolation history & $\begin{array}{c}\text { Orbit regularly varying } \\
\text { between perihelia near 1.5 } \\
\text { and 2.0 AU }\end{array}$ & Yeomans et al. (2004) & Steady non-gravitational forces \\
\hline
\end{tabular}




\section{Figure Captions}

Figure 1: R-band absolute magnitudes reduced to zero solar phase angle covering active and, possibly, inactive phases of comet 9P/Tempel 1. The phase law used is the polynomial shown in Table I (cf. Fig.4). These data, taken between 1997 and 2002 are part of the database generated during a world-wide observing campaign (Meech et al., 2004a) to support the Deep Impact mission. Beyond 4 AU the light curve is dominated by periodic variations associated with nucleus rotation and the mean brightness is essentially independent of heliocentric distance (cf. Fig.2 and 3). The horizontal dashed line is at $\mathrm{H}(\mathrm{R}, 1,1,0)=14.441$, the mean absolute magnitude at zero phase deduced by Hsieh and Meech (2004, and Table I). At distances closer to the sun than 3.5 AU the lightcurve begins to increase in brightness as a result of the volatization of surface materials and the development of a coma.

Figure 2: An expanded view of the data in Fig. 1 beyond 4 AU. It shows how the data are clustered in groups. So far, three of these, groups B, C, and D, have been used to estimate the rotational period. The horizontal dashed line is at $H(R, 1,1,0)=$ 14.441, the mean absolute magnitude at zero phase (Table I).

Figure 3. An expanded view of Group B ( $c f$. Fig. 2). The figure shows clearly how the underlying periodicity in the data is nearly commensurate with the diurnal sampling period. This makes the period of the comet and the magnitude range of the light curve difficult to pin down. The probable error of each point is \pm 
0.03 magnitudes. The photometric uncertainty in the individual data points is not shown to maintain the clarity of the figure. The horizontal dashed line is at $\mathrm{H}(\mathrm{R}, 1,1,0)=14.441$, the mean absolute magnitude at zero phase (Table I).

Figure 4. Dependence of absolute magnitude on solar phase angle, $\alpha$. Most of the apparent scatter of the points is due to rotational variations (typically $\sim 0.6 \mathrm{mag}$ peak-to-peak). The faint points near $\sim 12$ deg phase angle are an enigma. Possibly they are the result of irregularities in the shape of the nucleus casting large shadows near minimum light. The solid curve is for a sphere and was used to estimate the Hapke parameters $\left(\varpi_{0}=0.018, \mathrm{~h}=0.0135, \mathrm{~S}(0)=0.2, \mathrm{~g}=-\right.$ 0.525 , and $<\theta>=20 \mathrm{deg}$. (V-band)) The effective radius of the sphere needed for this fit was slightly larger $(3.43 \mathrm{~km})$ than for the model nucleus with its axial ratio of 3.2 and mean effective radius of $3.25 \mathrm{~km}$ (such dependencies of photometric parameters on shape have been discussed by Helfenstein and Veverka (1989)). The filled square is at $H(R, 1,1,0)=14.441$ the value found by Meech and Hsieh (2004) and the values of $\varpi_{0}, \mathrm{~S}(0)$, and $g$ are forced to be consistent with the V-band geometric albedo of 0.04 using a relationship developed by Hapke (1984). The color V-R $=0.56$ is assumed independent of phase angle in this analysis.

Figure 5. The data for group B has been phased to the rotational period of $41.85 \mathrm{hrs}$. The "saw-tooth" shape, double-peaked nature of the light curve and the possibility of unequal maxima and minima is clearly seen. Experience with this kind of 
light curve suggests an elongated nucleus with considerable irregularity. The error bars are the current estimate for the probable error of each point.

Figure 6. A WindowClean power spectrum (analogous to a periodogram) of the data in group B (cf. Fig.3). The main peak is at $1.20 \pm 0.08$ inverse days, i.e., a periodicity of $20 \pm 1.4 \mathrm{hrs}$. For a double peak lightcurve that is expected for an elongated object, this corresponds to a rotation period of $40 \pm 2.8 \mathrm{hrs}$. Preliminary analysis of groups B, C, and D together yields a rotation period of $41.85 \pm 0.1$ hrs (cf. Table I and Fig. 5).

Figure 7. A preliminary reduction of Hubble Space Telescope V-band data showing a double peaked lightcurve with considerable asymmetry. The magnitudes shown here are uncalibrated and represent the total light in the images. A much improved reduction that involves the extraction of the nucleus signal from any coma using knowledge of the point spread function is ongoing. The fit to the light curve is based on the provisionally adopted rotational period of $41.85 \mathrm{hr}$ and is the sum of the fundamental and the first two harmonics.

Figure 8. Normalized versions of the signal in the 2004 Spitzer and HST data combined in phase plots for periods at 39.6 and $41.85 \mathrm{hr}$ to show the sensitivity of the shape of the phased lightcurve with assumed rotation period. The $41.85 \mathrm{hr}$ period provides a smoother accounting of the combined lightcurve and is our currently 
adopted rotation period. A detailed assessment of these data, which includes an error analysis, is ongoing.

Figure 9. Determination of the rotation pole. The colored small circles represent the locus of the possible pole positions for an assumed value for the axial ratio of the model nucleus. Each color represents a separate group of observations taken under different geometrical conditions. The case for the axial ratio $(\mathrm{a} / \mathrm{b}=3.2)$, which gives the smallest spread in the locations of the intersections of the sky, is shown. Where the intersections of the small circles cluster together is a candidate position for the direction of the rotation axis on the sky. There are four such places that represent opposite ends of two photometrically equivalent poles. In Table I we quote only the directions that are in the Northern hemisphere and that are marked 1 and 2. The dashed lines that follow each small circle reflect the uncertainty in the observed amplitude of the lightcurve and help to better define the uncertainty in the pole solutions.

Figure 10. Visual observations of the telescopic appearance comet 9P/Tempel 1 made in April 1994 (left) and April 1983 (right). The geometric circumstances of the comet seen from Earth repeat almost exactly every 11 years. The similarity of the structures in the coma suggests that the rotational pole is reasonably stable and that we will likely see similar structures from Earth in April 2005. The existence of the structures suggests that only a small fraction of the nuclear surface is active. The drawing of the comet in 1983 was first published in the 
International Comet Quarterly and reprinted here by courtesy of J.-C. Merlin.

The drawing of the comet in 1994 is by courtesy of N. Biver. North is down and

East is to the right. Such drawings should eventually be helpful in locating active areas on the nucleus. 
Figure

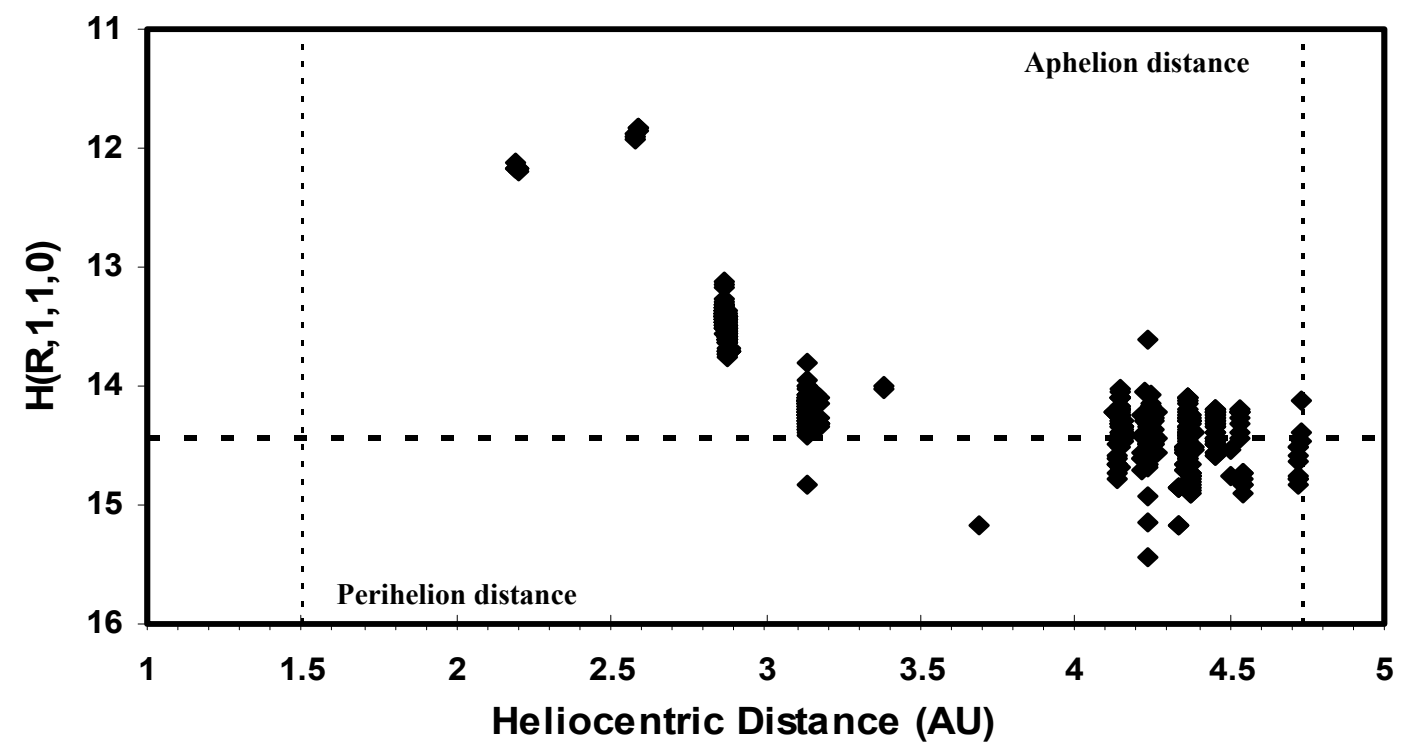


Figure 2

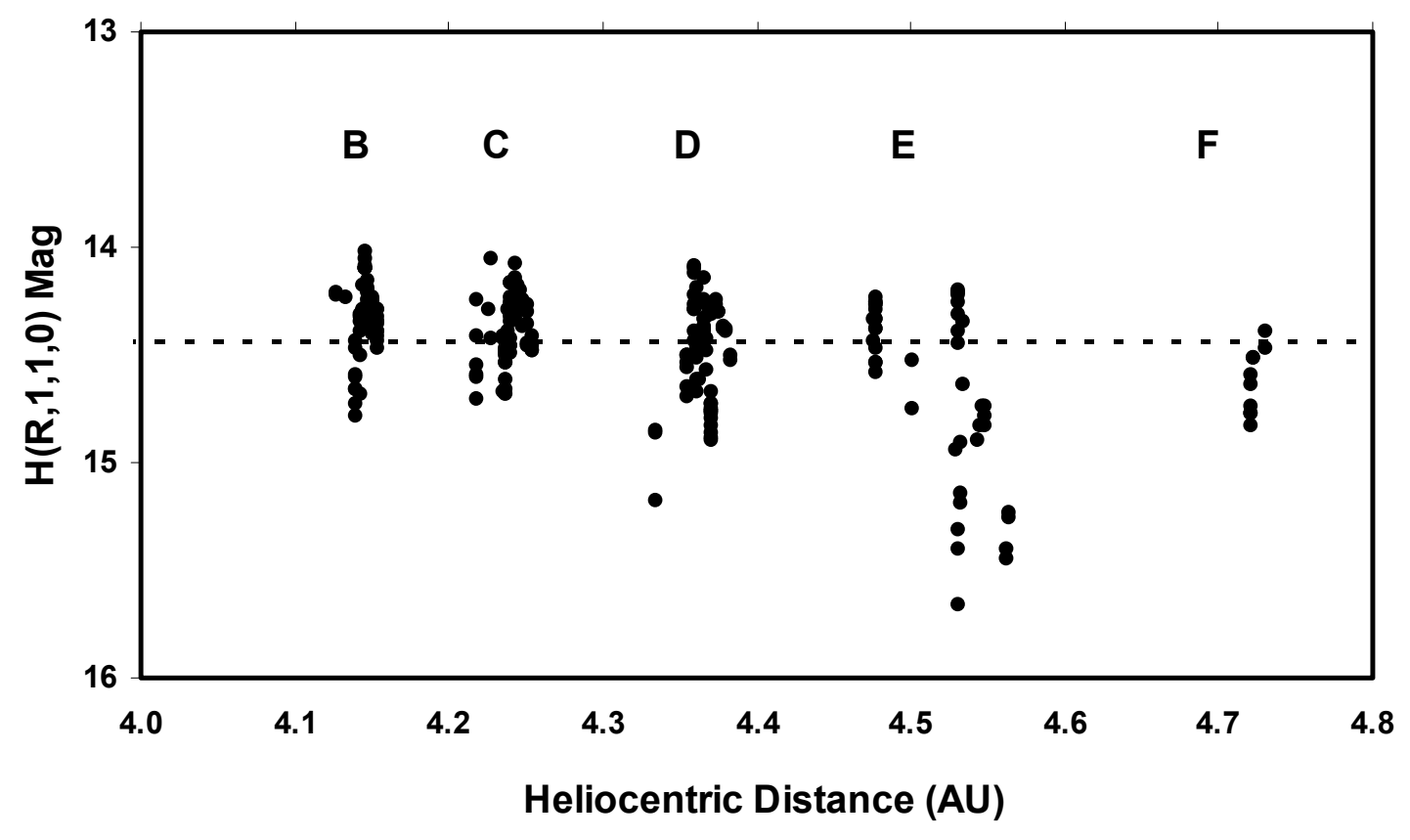


Figure 3

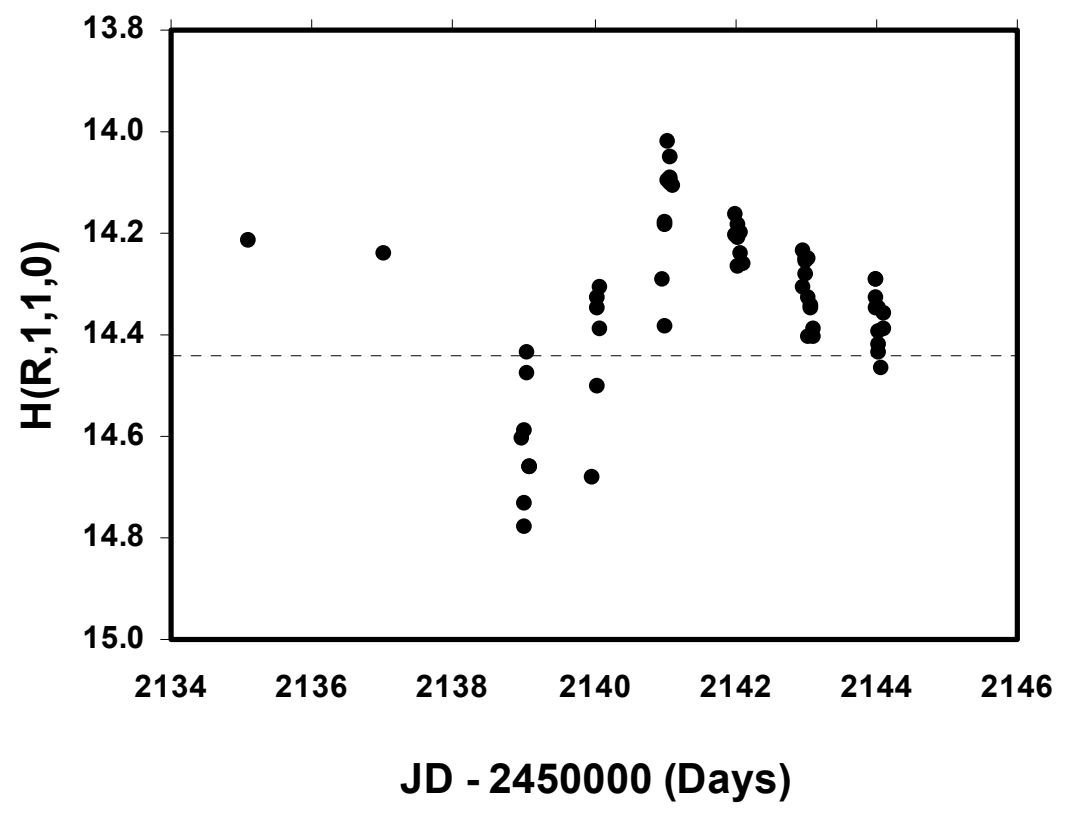


Figure 4.

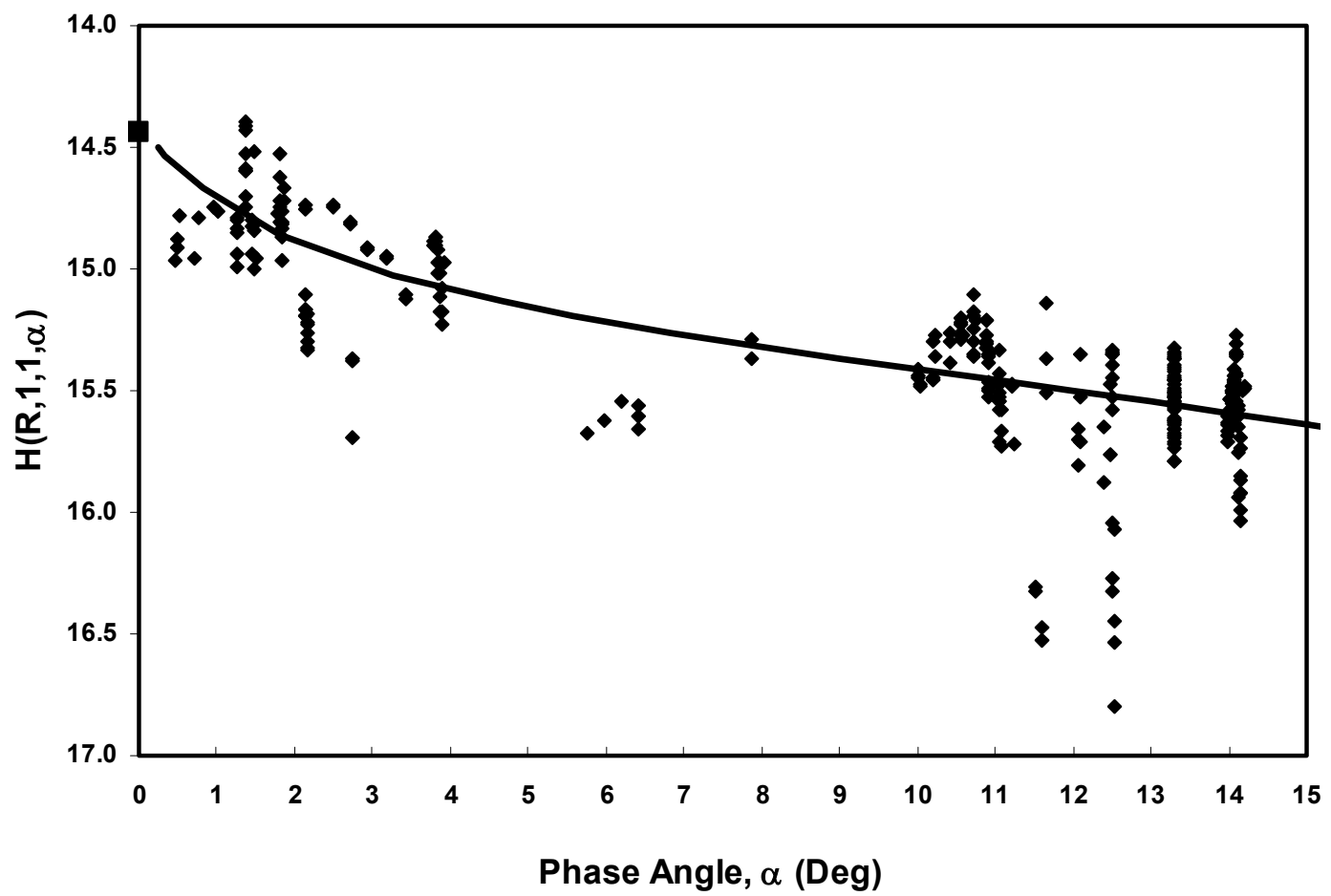


Figure 5

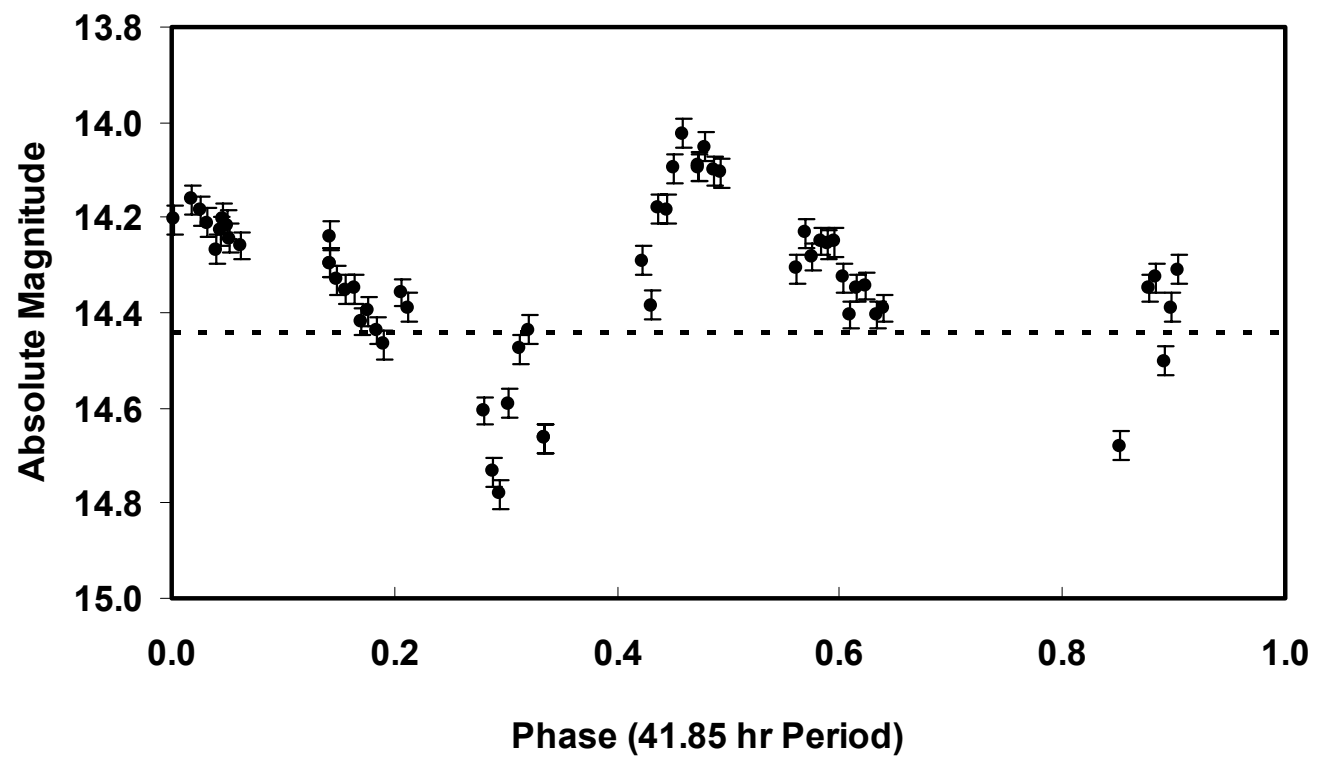


Figure 6.

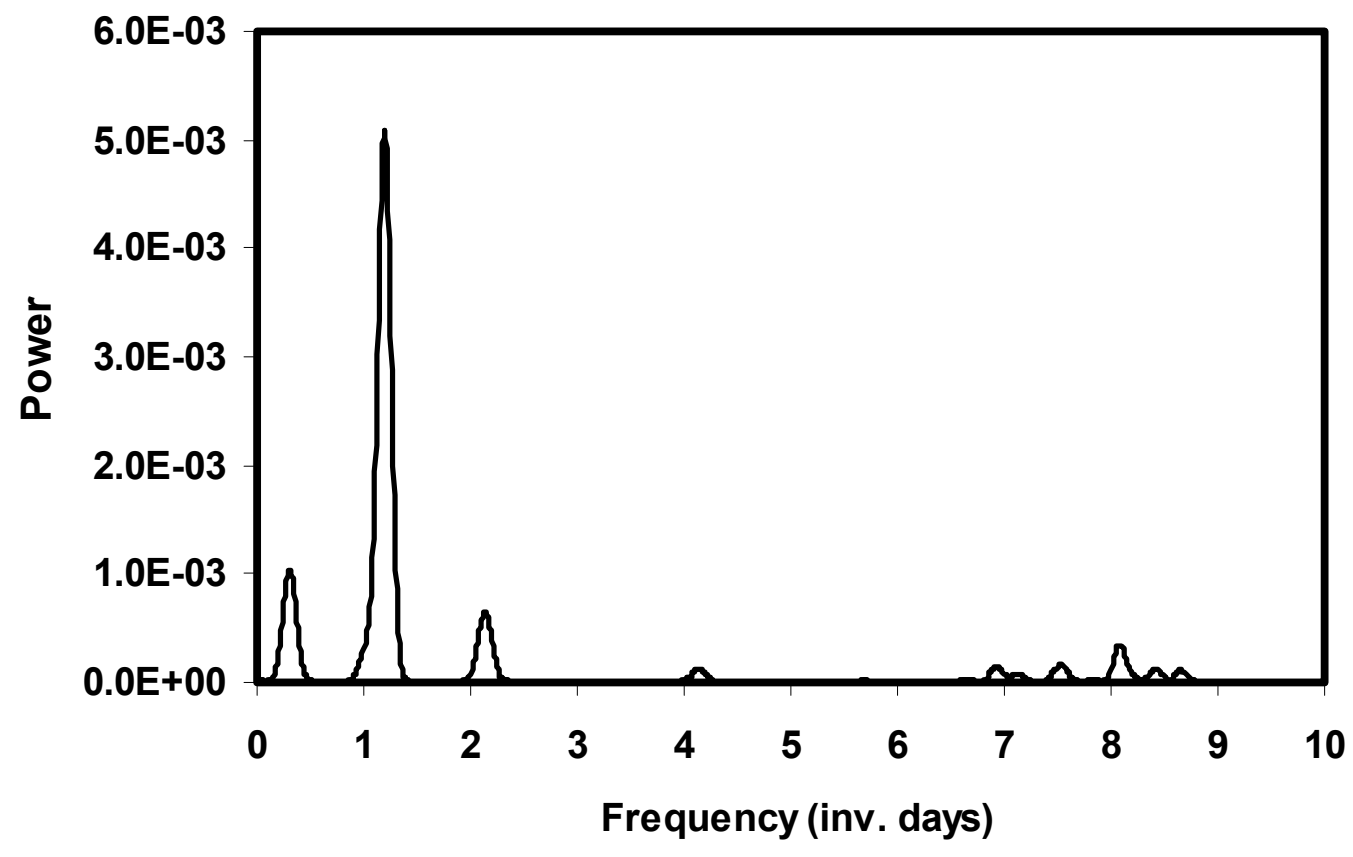


Figure 7

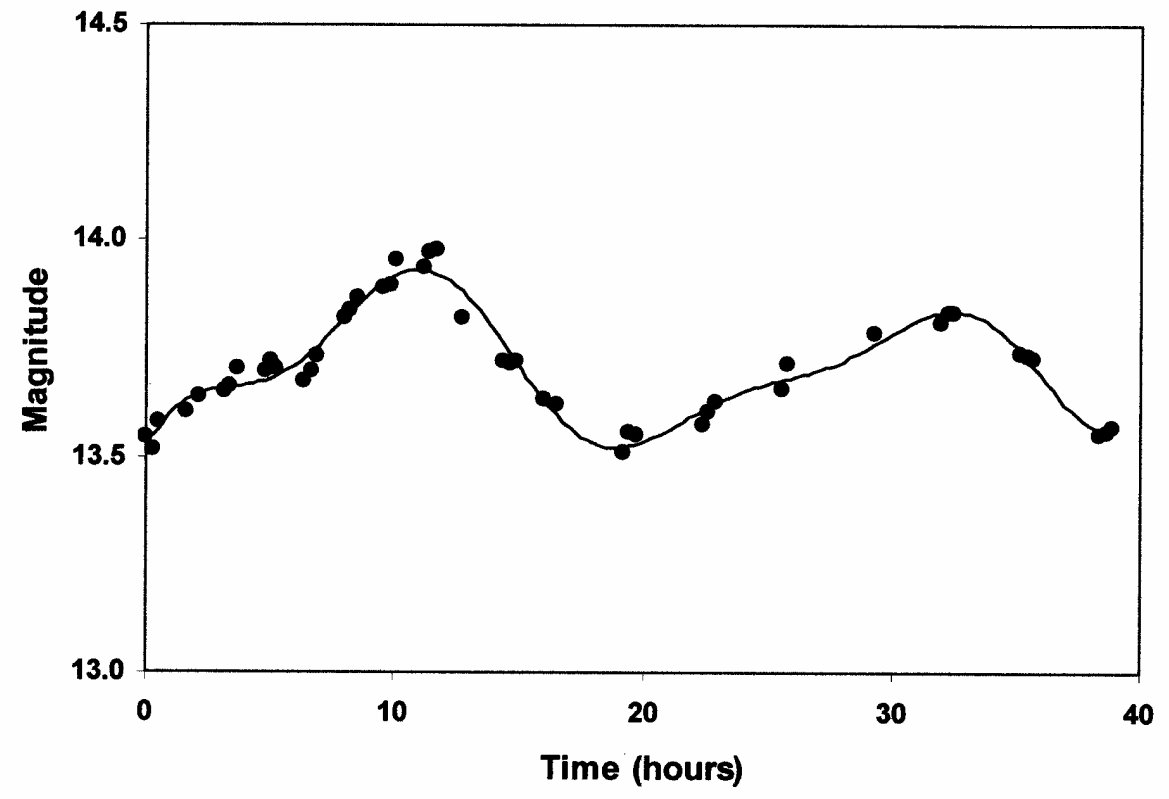


Figure 8
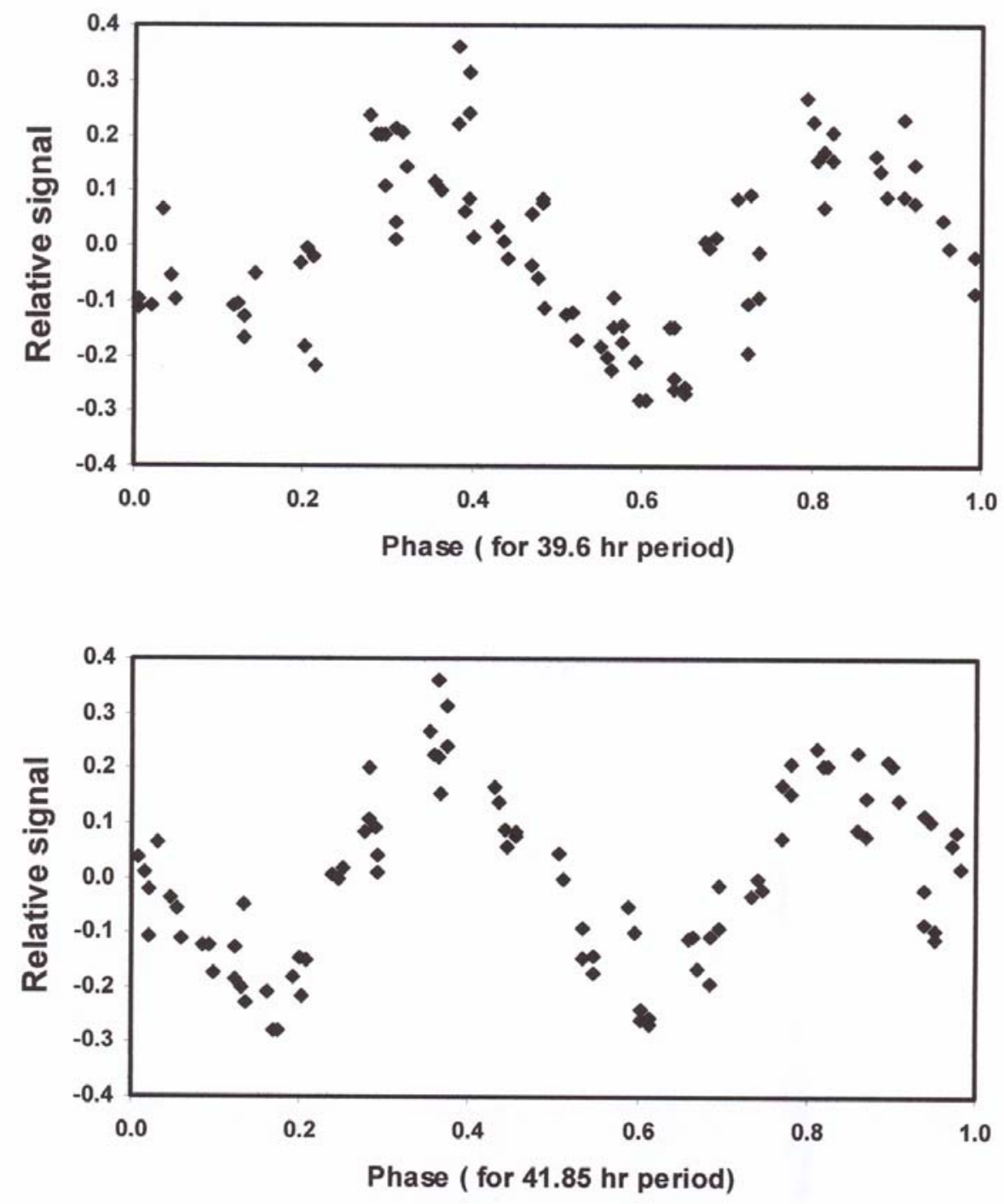
Figure 9

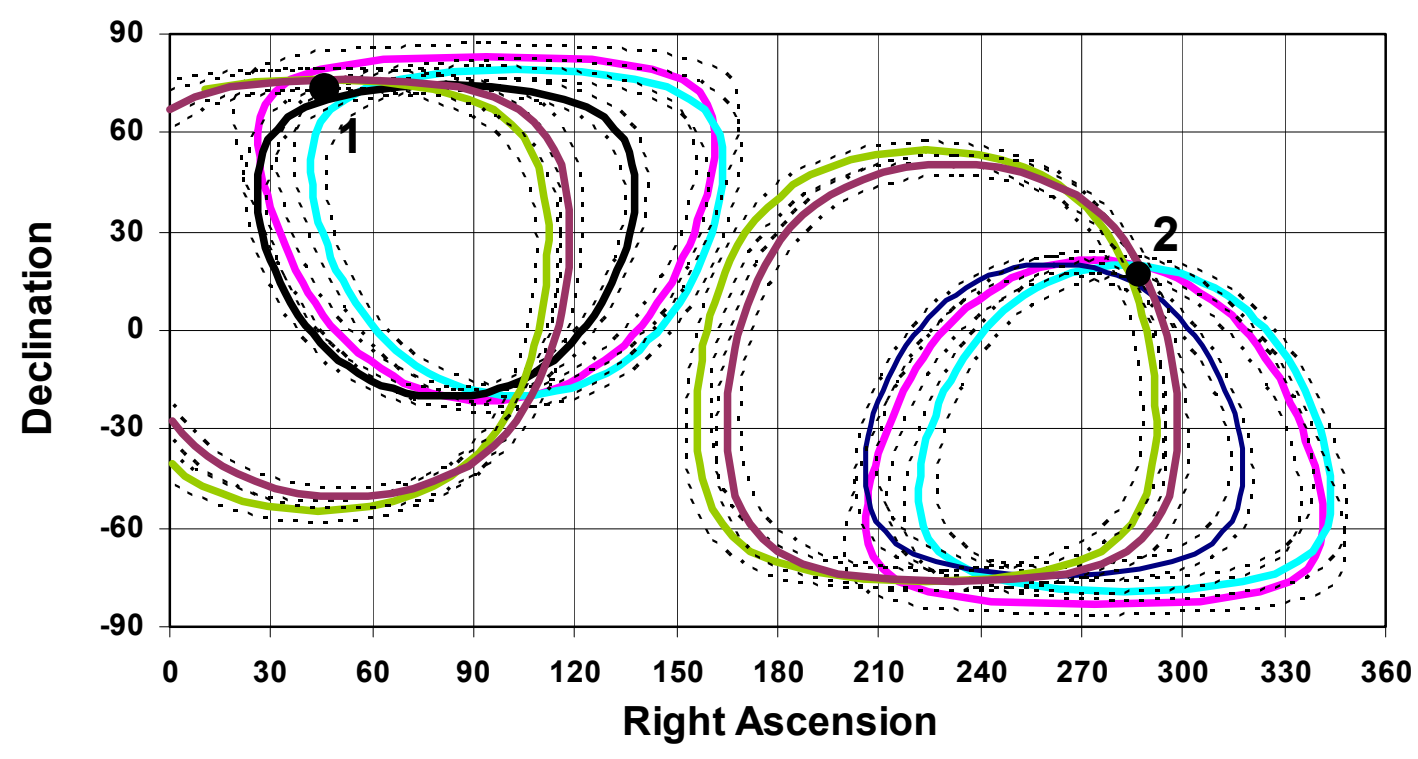


Figure 10
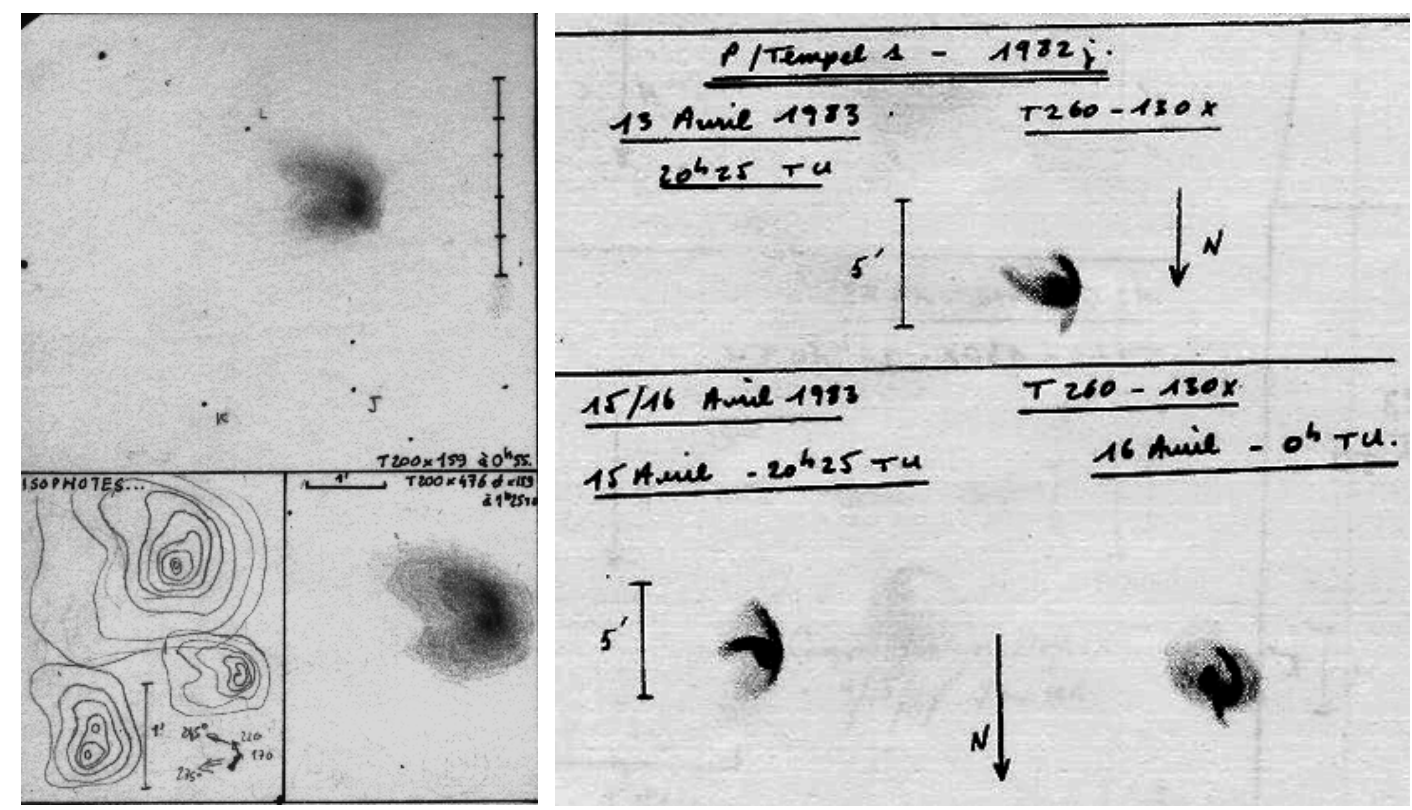